\title{
Efecto de diferentes niveles de cloruro de sodio sobre el rendimiento productivo de los pollos de engorde
}

\author{
Effect of different levels of sodium chloride on productive yield in broilers
}

\author{
S.C. Rubina ${ }^{1}$, H. N. Pujada , F. E. Airahuacho ${ }^{1,2}$
}

\begin{abstract}
Resumen
Objetivos: Evaluar el efecto de niveles de cloruro de sodio $(\mathrm{NaCl})$ del agua de bebida en el rendimiento productivo de los pollos de engorde. Metodología: Se suministró agua con tres niveles de $\mathrm{NaCl}$ $(0 ; 0,075$ y $0,125 \%)$ a pollos desde los 11 hasta los 31 días de edad con la finalidad de evaluar su efecto sobre el peso vivo (PV), consumo de alimento (CA), conversión alimenticia (CAL) e ingestión de agua (IA). Los niveles de cloruro de sodio fueron diluidos en agua destilada y se utilizaron bebederos niples para hacer más precisa la medición del consumo de agua. Resultados: Niveles de $0,075 \%$ de $\mathrm{NaCl}$ en el agua de bebida mejoraron el PV, CA, CAL y la IA, mientras que el nivel de $0,125 \%$ $\mathrm{NaCl}$ disminuyó el rendimiento productivo y aumentó el consumo de agua. Los requerimientos de $\mathrm{Na}$ y $\mathrm{Cl}$ adecuados, considerando la ingesta total de ambos minerales, serían de $0,243 \%$ y $0,279 \%$, respectivamente. Conclusiones: La inclusión de cloruro de sodio en el agua de bebida influyó sobre el rendimiento productivo del pollo de carne, observándose rendimientos satisfactorios con la inclusión de $0,075 \% \mathrm{NaCl}$ en el agua de bebida.
\end{abstract}

Palabras clave: Cloruro de sodio, sodio, cloro, pollos de engorde, agua

\begin{abstract}
Objectives: To evaluate the effect of sodium chloride $(\mathrm{NaCl})$ levels in drinking water on productive yield in broilers. Methodology: Water with three levels of $\mathrm{NaCl}(0 ; 0.075$ and $0.125 \%)$ was supplied to chickens from 11 to 31 days of age in order to evaluate its effect on live weight (PV), feed consumption (CA), food conversion (CAL) and water intake (IA). The sodium chloride levels were diluted in distilled water and nipple drinkers were used to make the measurement of water consumption more accurate. Results: $0.075 \% \mathrm{NaCl}$ levels in the drinking water improved the PV, CA, CAL and AI, while the $0.125 \% \mathrm{NaCl}$ level decreased productive yield and increased water consumption. The adequate $\mathrm{Na}$ and $\mathrm{Cl}$ requirements, considering the total intake of both minerals, would be $0.243 \%$ and $0.279 \%$ respectively. Conclusions: The inclusion of sodium chloride in the drinking water influenced the productive yield of the meat chicken, observing satisfactory yield with the inclusion of $0.075 \% \mathrm{NaCl}$ in the drinking water.
\end{abstract}

Keywords: sodium chloride, sodium, chlorine, broilers, water

\section{Introducción}

El agua de bebida varía ampliamente en su contenido de sales y otros minerales, desde el agua que contiene muy poca sal hasta algunas altamente salinas. La sal puede ser una fuente valiosa de minerales en las zonas con consumos reducidos de sodio en el alimento o resultar tóxica por la ingestión de cantidades excesivas de sal o de otros minerales (Underwood \& Suttle, 2003).

\footnotetext{
${ }^{1}$ Universidad Nacional José Faustino Sánchez Carrión, Lima,Perú.

22Autor para correspondencia: fairahuacho@unjfsc.edu.pe

48
} 
El sodio (Na) y cloro $(\mathrm{Cl})$ son macro minerales esenciales para el metabolismo animal y se suplementan en dietas en forma de cloruro de sodio $(\mathrm{NaCl})$ (Mcdonald et al., 2010). Ambos mantienen la presión osmótica, el equilibrio ácido básico, controlan el paso de nutrientes a las células y el metabolismo del agua, proteínas, energía y minerales (Patience, 1990; Underwood \& Suttle, 2003). La deficiencia de Na reduce la absorción de aminoácidos y monosacáridos en el tracto gastrointestinal (Guyton, 2016). La homeostasis de los fluidos corporales está relacionada con la ingesta, retención y excreción de $\mathrm{Na}$ (González, 2013), y para mantener la homeostasis orgánica su ingesta debe ser adecuada (Pinheiro et al., 2011). Con respecto al $\mathrm{Cl}$, dietas altas con este mineral disminuyen el pH de la sangre en los pollos (Borges et al., 2003) y su deficiencia causa trastornos nerviosos. En ambos escenarios, afectan el crecimiento animal (Underwood \& Suttle, 2003; Pinheiro et al., 2011).

Las necesidades de $\mathrm{Na}$ (así como $\mathrm{K}$ y $\mathrm{Cl}$ ) para aves en crecimiento suelen disminuir a medida que se acercan al peso adulto. Las primeras investigaciones reportan que las necesidades de $\mathrm{Na}$ disminuían de 1,3 a 0,7 g Na/kg $\mathrm{MS}$ entre la 1ra - 3ra y 7ma - 9na semana de edad (Borges et al., 2003), investigaciones posteriores recomiendan entre 4 - $5 \mathrm{~g} \mathrm{Na} / \mathrm{kg}$ MS durante la primera semana, disminuyendo a $3 \mathrm{~g} \mathrm{Na} / \mathrm{kg} \mathrm{MS}$ después de la segunda semana de edad (OviedoRondón et al., 1991). Las bajas necesidades recomendadas actualmente en diferentes tablas nutricionales se debe a la presencia de $\mathrm{Na}$ en el agua de bebida (Underwood \& Suttle, 2003). Rostagno et al. (2017) recomiendan necesidades que varían desde 0,225 a 0,192\% $\mathrm{Na}$ en la ración para pollos de engorde machos entre 01 a 46 días de edad; mientras que en las normas de la Fundación española para el desarrollo de la nutrición animal (Santomá y Mateos, 2018) sus recomendaciones mínimas varían entre 0,19 a $0,15 \%$ Na para pollos de carne de 01 a 37 días de edad.

A pesar de la vital importancia de este mineral, existen pocas investigaciones sobre sus requerimientos nutricionales reales y se relacionaría a que las fuentes suplementarias comúnmente utilizadas (cloruro de sodio o bicarbonato de sodio) tienen un precio relativamente bajo (Barros et al., 2001). El exceso de la ingesta de sales minerales determina un incremento en la excreción urinaria, aumento en el consumo de agua, aumento de cama húmeda, trastornos del tejido plantar $\mathrm{y}$ deterioro del rendimiento avícola (Bondi, 1988). Este problema puede mitigarse en parte, reduciendo la cantidad de cloruro de sodio $(\mathrm{NaCl})$ en el alimento. Por ello, el objetivo del estudio fue evaluar el efecto de los niveles de $\mathrm{NaCl}$ en el agua sobre el rendimiento productivo de pollos de engorde.

\section{Metodología}

La investigación se realizó en el galpón experimental de aves de la Escuela Profesional de Ingeniería Zootécnica, ubicado en distrito de Huacho; provincia, Huaura; región Lima provincias. Se empleó 54 pollos machos Cobb de 11 días de edad, que fueron agrupados al azar en tres tratamientos y distribuidos en 18 unidades experimentales de tres pollos cada uno. El tratamiento control $\left(\mathrm{T}_{0}\right)$ recibió sólo agua desionizada, $0 \% \mathrm{NaCl}$. Los tratamientos experimentales consumieron dos niveles de $\mathrm{NaCl}, \mathrm{T}_{1}: 0,075 \%$ y $\mathrm{T}_{2}: 0,125 \%$. El agua fue suministrado a voluntad en bebederos niples. Las variables evaluadas fueron el peso vivo (PV), consumo de alimento (CA), conversión alimenticia (CAL) e ingestión de agua (IA). Las dietas fueron balanceadas por programación lineal al mínimo costo $\mathrm{y}$ utilizando las recomendaciones nutricionales de la línea comercial (Tabla 1). Se realizó el análisis de variancia, para determinar diferencias entre tratamientos y la prueba de Tukey para determinar diferencias entre los promedios de los parámetros evaluados.

\section{Resultados y discusión}

El efecto de los niveles de $\mathrm{NaCl}$ sobre el PV, CA, CAL e ingestión de agua y minerales, desde los 11 hasta los 31 días de edad, se muestran en la Tabla 2 .

Se encontraron diferencias significativas entre los parámetros productivos evaluados $(P<0,05)$. El $\mathrm{T}_{1}(0,075 \% \mathrm{NaCl})$ mostró un 
Tabla 1

Composición porcentual y valor nutritivo estimado de las dietas experimentales

\begin{tabular}{lcc}
\hline \multirow{2}{*}{ Ingredientes } & \multicolumn{2}{c}{ Dietas } \\
\cline { 2 - 3 } & $11-22$ días & 23 - 31 días \\
\hline Aceite de Soya & 0,68 & 3,47 \\
Maíz & 71,00 & 58,53 \\
Harina de pescado & 5,76 & - \\
Torta de soya & 19,29 & 17,54 \\
Soya Integral & - & 10,00 \\
Afrecho & - & 6,22 \\
Lisina HCl & 0,13 & 0,16 \\
DL-Metionina & 0,17 & 0,22 \\
Fosfato Dicálcico & 1,35 & 1,96 \\
Carbonato de calcio & 1,01 & 1,03 \\
Cloruro de sodio & 0,31 & 0,09 \\
Bicarbonato de sodio & - & 0,50 \\
Coccidiostato & 0,05 & 0,05 \\
Premix & 0,15 & 0,15 \\
Cloruro de colina & 0,05 & 0,05 \\
Promotor de crecimiento & 0,05 & 0,05 \\
\hline Contenido Nutricional & & \\
\hline Energía Met., Mcal/kg & 3,083 & 3,190 \\
Proteína cruda, \% & 19,0 & 18,7 \\
Fibra, \% & 3,00 & 3,33 \\
Lisina, \% & 1,15 & 1,05 \\
Met + cist., \% & 0,92 & 0,83 \\
Treonina, \% & 0,80 & 0,73 \\
Calcio, \% & 0,96 & 0,89 \\
Fósforo disponible, \% & 0,48 & 0,44 \\
Sodio, \% & 0,19 & 0,19 \\
Cloro, \% & 0,30 \\
\hline
\end{tabular}

mayor PV que el $\mathrm{T}_{2}(0,125 \% \mathrm{NaCl})$ y similar al $\mathrm{T}_{0}(0 \% \mathrm{NaCl})$. La mejora en el incremento de PV con el aumento de Na nutricional aparentemente está determinada por el mayor consumo de alimento (Penz, 2003), tal como ocurre en el presente estudio con las aves que consumieron agua con $0,075 \% \mathrm{NaCl}$. El efecto negativo de elevados niveles de $\mathrm{Na}$ sobre el crecimiento se relacionaría con un mayor gasto de energía de la bomba sodio-potasio, como una forma de controlar el gradiente electroquímico entre el medio extra e intracelular, o la toxicidad del mineral en altos niveles (Barros et al., 2001).
Tabla 2

Efecto del cloruro de sodio en el agua sobre la ganancia de peso, consumo de alimento y conversión alimenticia en pollos de engorde desde los 11 hasta los 31 días de edad

\begin{tabular}{lccc}
\hline & \multicolumn{3}{c}{ Tratamientos } \\
\cline { 2 - 4 } $\begin{array}{c}\text { Parámetros } \\
\text { evaluados }\end{array}$ & $\mathrm{T}_{0}$ & $\mathrm{~T}_{1}$ & $\mathrm{~T}_{2}$ \\
\cline { 2 - 4 } & $0 \%$ & $0,075 \%$ & $0,125 \%$ \\
& $\mathrm{NaCl}$ & $\mathrm{NaCl}$ & $\mathrm{NaCl}$ \\
\hline Peso inicial, g & 261 & 272 & 262 \\
Peso final, g & $1559^{\mathrm{ab}}$ & $1612^{\mathrm{a}}$ & $1536^{\mathrm{b}}$ \\
$\begin{array}{l}\text { Consumo de } \\
\text { alimento, g }\end{array}$ & $2909^{\mathrm{b}}$ & $3001^{\mathrm{a}}$ & $3014^{\mathrm{a}}$ \\
$\begin{array}{l}\text { Conversión } \\
\text { alimenticia }\end{array}$ & $2,24^{\mathrm{a}}$ & $2,24^{\mathrm{a}}$ & $2,37^{\mathrm{b}}$ \\
$\begin{array}{l}\text { Ingestión de } \\
\text { agua,ml }\end{array}$ & $4989^{\mathrm{c}}$ & $5522^{\mathrm{a}}$ & $5425^{\mathrm{b}}$ \\
\hline $\begin{array}{l}\text { a,b,c } \text { Letras distintas entre filas indican diferencia estadistica } \\
(P<0,05) .\end{array}$
\end{tabular}

Las aves que consumieron agua con mayores niveles de cloruro de sodio mostraron un mayor consumo de alimento $(P<0,05)$. El mayor consumo de alimento de las aves del tratamiento con $0,075 \% \mathrm{NaCl}(0,049 \% \mathrm{Na})$ se vio reflejado en un mayor PV y una mejor CAL. Penz (2003) refiere que la mejora en el aumento de peso con el aumento de Na nutricional aparentemente está determinada por la mayor ingesta de alimento, tal como ocurre con las aves que consumieron agua con $0,075 \% \mathrm{NaCl}$. Sin embargo, este resultado no concuerda con el obtenido por Murakami et al. (1997) quienes observaron que la ingesta de alimento era influenciada significativamente por el contenido de $\mathrm{Cl}$, y no por el contenido de $\mathrm{Na}$ en el alimento.

En el presente estudio, niveles de $0,125 \% \mathrm{NaCl}$ $(0,049 \% \mathrm{Na})$ en el agua afectó negativamente el crecimiento y conversión alimenticia de los pollos, estos resultados difieren de los obtenidos por Barros et al. $(2001,2004)$ quienes concluyeron que con niveles de $0,256 \%$ de $\mathrm{Na}$ en la dieta reportaron incremento en la ganancia de peso y conversiones alimenticias eficientes en pollos de engorde de 1 a 42 días de edad. En codornices de 1 a 21 días, el nivel de $\mathrm{Na}$ tuvo un 
efecto lineal sobre la ingesta de alimento y agua pero un efecto cuadrático sobre el aumento de peso y la conversión alimenticia, siendo $0,23 \mathrm{y}$ $0,21 \% \mathrm{Na}$ los mejores niveles. Sin embargo, en la fase de 21 a 42 días, el aumento en el nivel de Na no influyó en la ingesta de alimento, sino que promovió una reducción lineal en el aumento de peso, un aumento en el consumo de agua y un empeoramiento en la conversión del alimento (Lima et al., 2011).

Una mayor ingesta de agua con la provisión de altos niveles de $\mathrm{Na}$ en el alimento está relacionada con la necesidad de las aves de mantener la homeostasis corporal (Barros et al., 2004; Borges et al., 2003). A medida que aumenta la ingesta de $\mathrm{Na}$, aumenta la osmolaridad de la sangre $\mathrm{y}$, a través de mecanismos neurohormonales, se desarrolla la sensación de sed, que estimula el consumo de agua por parte del ave para excretar el exceso de Na (González, 2013). En consecuencia, este mayor consumo también está relacionado con la ingesta de alimento, la edad y el peso de las aves, la temperatura del agua y el $\mathrm{pH}$ (Raquel et al., 2011). Murakami et al. (2000) y Silva et al. (2006) mencionan que el efecto del aumento del nivel de $\mathrm{Na}$ en la alimentación de pollos de engorde conduce a una mayor humedad de la camada debido a una mayor humedad de las excretas como consecuencia de una mayor ingesta de agua. Según lo descrito por Borges et al. (2003), la humedad de la cama es un reflejo de la ingesta de agua del ave.

En el presente estudio, la presencia de $\mathrm{NaCl}$ en el agua de bebida en pollos de engorde de 11 a 31 días de edad influyó sobre los parámetros productivos. Considerando la ingesta total de ambos minerales, las necesidades adecuadas de $\mathrm{Na}$ y $\mathrm{Cl}$ serían de $0,243 \%$ y $0,279 \%$ respectivamente (Tabla 3) que es superior al obtenidos por Borges et al. (2003), quienes determinaron que con un nivel de $0,197 \%$ de $\mathrm{Na}$ en la dieta alimenticia para pollos de engorde se lograron mejores rendimientos productivos. Estos resultados sugieren que niveles de 0,075\% $\mathrm{NaCl}$ en el agua complementaria el $\mathrm{Na}$ y $\mathrm{Cl}$ dietario y cubriría las necesidades de las aves de estos minerales; mientras que el nivel de $0,125 \% \mathrm{NaCl}$ excedería las necesidades del ave, conllevando a una disminución del crecimiento, aumento en el consumo de alimento, deterioro de la conversión alimenticia, y aumento de la ingesta de agua.

\section{Tabla 3}

Ingestión total de $\mathrm{Na}$ y $\mathrm{Cl}$ en el pollo de engorde desde los 11 hasta los 31 días de edad

\begin{tabular}{lccc}
\hline & \multicolumn{3}{c}{ Tratamientos } \\
\cline { 2 - 4 } Ingestión & $0 \%$ & $0,075 \%$ & $0,125 \%$ \\
& $\mathrm{NaCl}$ & $\mathrm{NaCl}$ & $\mathrm{NaCl}$ \\
\hline Ingestión de Na del alimento, g & 5526 & 5702 & 5726 \\
Ingestión de Cl del alimento, g & 5693 & 5876 & 5910 \\
Ingestión de Na del agua, g & 0 & 1601 & 2658 \\
Ingestión de Cl del agua, g & 0 & 2485 & 4069 \\
Ingestión total de Na/ave, g & 5526 & 7303 & 8384 \\
Ingestión total de Cl/ave, g & 5693 & 8361 & 9979 \\
Sodio dietario, \% & 0,190 & 0,243 & 0,278 \\
Cloro dietario, \% & 0,196 & 0,279 & 0,331 \\
\hline
\end{tabular}

\section{Referencias}

Barros, J.M.S., Gomes, P.C., Albino, L.F.T., Rostagno, H.S., \& Costa, L.F. (2004). Exigência de sódio para frangos de corte nas fases de crescimento (22 a 42 dias) e final (43 a 53 dias). Revista Brasileira de Zootecnia, 33(6, Suppl. 1), 1721-1733. doi. org/10.1590/S1516-35982004000700011

Barros, J.M.S., Gomes, P.C., Rostagno, H.S., Albino, L.F.T., \& Nascimento, A.H. (2001). Exigência nutricional de sódio para frangos de corte de 1 a 21 dias de idade. Revista Brasileira de Zootecnia, 30(3, Suppl. 1), 1044-1051. doi.org/10.1590/S151635982001000400019

Bondi, A.A. (1988). Nutrición Animal. Zaragoza, España: Acribia S.A.

Borges, S.A., Maiorka, A., \& Silva, A.V.F. (2003). Fisiologia do estresse calórico e a utilização de eletrólitos em frangos de corte. Ciência Rural, 33(5),975-981. doi.org/10.1590/ S0103-84782003000500028

Oviedo-Rondón, E.O., Murakami, A.E., Furlan, A.C., Moreira, I. \& Macari, M. (1991). 
Sodium and Chloride Requirements of Young Broiler Chickens Fed Corn-Soybean Diets (One to Twenty-One Days of Age). Poultry Science, 70,1-18. doi.org/10.1093/ ps/80.5.592

Guyton, A.C \& Hall, J.E. (2016). Tratado de fisiología médica. Recuperado de: http:// www.untumbes.edu.pe/bmedicina/libros/ Libros10/libro125.pdf

Lima, R.C., Freitas, E.R., Raquel, D.L, Sá, N.L., Lima, C.A., \& Paiva, A.C. (2011). Níveis de sódio para codornas japonesas na fase de crescimento. Revista Brasileira de Zootecnia, 40(2), 352-360. doi.org/10.1590/ S1516-35982011000200017

González, J.F. (2013, junio). Efectos del agua en la salud y producción avícola. Plumazos, (44),23-28. Recuperado de: https://www. researchgate.net/publication/264084094 Efectos_de_la_calidad_del_agua_en_ salud_y_produccion_avicola

McDonald, P., Edwards, L. A., Greenhalgh, J. F. D., Morgan, C. A., Sinclair, L. A. \& Wilkinson, R. G. (2010). Animal Nutrition, Seventh Edition. London, UK: Pearson.

Murakami, A.E., Watkins, S.E., Saleh, E.A., England, J.A. \& Waldroup, P.W. (1997). Estimation of the sodium and chloride requirements for the young broiler chick. Journal of Applied Poultry Research, 6(1),155-162. doi.org/10.1093/japr/6.2.155

Patience, J.F. (1990). A review of the role acid-base balance in amino acid nutrition. Journal Animal Science, 68, 398-408. doi.org/10.2527/1990.682398x

Penz, A. M. (2003). Importância da água na produção de frangos de corte. En T. M. Biavatti (Ed.), Anais do IV Simpósio Brasil Sul de Avicultura (pp. 112-131). Chapecó, Brasil: EMBRAPA.
Pinheiro, S.R.F., Sakomura, N.K., Kawauchi, I.M., Bonato, M.A., Dorigam, J.C.P., \& Fernandes, J.B.K. (2011). Sodium chloride levels for Colonial broiler chickens reared in free-range system. Revista Brasileira de Zootecnia, 40(7), 1545-1553. doi. org/10.1590/S1516-35982011000700021

Raquel, D.L., Lima, R.C., Freitas, E.R., Nascimento, G.A.J., Sá, N.L. \& Paiva, A.C. (2011). Níveis de sódio para codornas italianas destinadas à produção de carne. Revista Brasileira de Zootecnia, 40(1), 135-141. doi.org/10.1590/S151635982011000100019

Rostagno, H. S. Teixeira, L.F., Hannas, M.I., Donzele, J.L., Sakomura, N.K., Perazzo, F.G., Saraiva, A., Teixeira, M.L., Rodrigues, P.B., Oliveira, R.F., Toledo, S.L. \& Oliveira, C. (2017). Tablas brasileñas para aves y cerdos: composición de alimentos y requerimientos nutricionales. Recuperado de: https://bit.ly/2EO9xI8

Santomá, G. \& Mateos, G.G. (2018). Necesidades nutricionales para la avicultura: Normas FEDNA. Segunda edición, Madrid, España. Editorial Fundación Española para el desarrollo de la nutrición animal. Recuperado de : http:// www.fundacionfedna.org/sites/default/files/ NORMAS FEDNA AVES 2018v.pdf

Silva, J.D.B.S., Fuentes, M.F.F., Freitas, E.R., Espíndola, G.B., Sousa, F.M. \& Cruz, C.E.B. (2006). Níveis de sódio em rações de pintos de corte na fase inicial. Revista Ciência Agronômica, 37 (1),84-90.

Underwood, E., Suttle, N.F. (2003). Los Minerales en la nutrición del ganado, $3^{\circ} \mathrm{ed}$. Zaragoza, España: Acribia S.A. 